\title{
E-beam nano-patterning for the ordered growth of GaN/InGaN nanorods
}

\author{
Francesca Barbagini , Ana Bengoechea-Encabo , Steven Albert , Pierre Lefebvre , Javier Martinez , \\ Miguel Angel Sanchez-Garcia , Achim Trampert , Enrique Calleja
}

\section{A R T I I C L E I I N F O}

\section{Keywords:}

E-beam lithography

Nitride nanorods

Ordered growth

\begin{abstract}
A B S T R A C T
E-beam lithography was used to pattern a titanium mask on a GaN substrate with ordered arrays of nanoholes. This patterned mask served as a template for the subsequent ordered growth of GaN/InGaN nanorods by plasma-assisted molecular beam epitaxy. The mask patterning process was optimized for several holes configurations. The smallest holes were $30 \mathrm{~nm}$ in diameter with a pitch (center-to-center distance) of $100 \mathrm{~nm}$ only. High quality masks of several geometries were obtained that could be used to grow ordered GaN/InGaN nanorods with full selectivity (growth localized inside the nanoholes only) over areas of hundreds of microns. Although some parasitic InGaN growth occurred between the nanorods during the In incorporation, transmission electron microscopy and photoluminescence measurements demonstrated that these ordered nanorods exhibit high crystal quality and reproducible optical properties.
\end{abstract}

\section{Introduction}

III-Nitride nanorods (NRs) grown by molecular beam epitaxy (MBE) are known for their excellent crystal quality and optoelectronic properties [1,2]. For this reason, they have been investigated in the last few years for the fabrication of state-of-the-art nano-photodetectors, light-emitting diodes, and solar cells [3-6]. MBE NRs are commonly grown in the self-assembled conditions [7]. However, self-assembled NRs are characterized by inhomogeneous size and distribution that affect the electronic properties of the final device and render its processing very difficult. Recently, the selective area growth (SAG) method was introduced, where the NRs selectively grow in an ordered way on pre-defined sites on pre-patterned substrates [8]. SAG NRs exhibit homogeneous size, excellent geometrical ordering, and optimal opto-electronic properties.

The SAG approach requires that the substrate is patterned with arrays of ordered nanoholes, which serve as preferential sites for NRs nucleation. In this work, a titanium mask deposited on GaN substrates was patterned with nanoholes by using e-beam lithography (EBL) and reactive ion etching. This patterned mask was subsequently used to grow GaN/InGaN NRs in the SAG mode by plasma-assisted (PA) MBE. By varying the e-beam exposure parameters (current, time) and the thickness of the photoresist, we could achieve highly ordered nanoholes with diameter and pitch down to

\footnotetext{
* Corresponding author.

E-mail address: fbarbagini@isom.upm.es (F. Barbagini).
}

$30 \mathrm{~nm}$ and $100 \mathrm{~nm}$, respectively. Subsequent MBE growth lead to perfectly ordered crystalline GaN NRs that nucleate and grow with $100 \%$ selectivity inside the nanoholes, independently of the particular mask geometry, and over areas of hundreds of microns. In a second set of samples, an InGaN nano-disk with approximate size $10-20 \mathrm{~nm}$ was imbedded in the GaN NR. Although some parasitic growth of InGaN between the NRs occurred during this stage, photo-luminescence spectra of these samples revealed an intense light-blue emission that was attributed to this nano-disk. The high precision and reproducibility of e-beam patterning resulted in homogeneous size and geometry for the subsequently grown NRs that yielded intense and highly reproducible optical emission.

\section{Experimental}

Ten nanometers thick Ti films were deposited by e-beam evaporation onto commercial 2 inch-wafers consisting of $4 \mu \mathrm{m}$ GaN (0001) on sapphire (Lumilog, France), surface roughness $0.4 \pm 0.1 \mathrm{~nm}$ over $1 \mu \mathrm{m}^{2}$ (RMS). For the EBL patterning, a diluted resist (ZEP520A) was spun on the Ti film. After baking at $190^{\circ} \mathrm{C}$ for $2 \mathrm{~min}$, the holes were opened using a current of $0.5 \times 10^{-9} \mathrm{~A}$ and exposure times varying from 15 to $45 \mu$ s. The sample was subsequently etched in $\mathrm{O}_{2} / \mathrm{CF} 4$ plasma at $10^{-5}$ bar and $110 \mathrm{~W}$ (Plasmalab. Oxford Instr., UK) for $130 \mathrm{~s}$. The post-etch residue was removed by immersing in pyrrolidone at $80^{\circ} \mathrm{C}$ for $30 \mathrm{~min}$ and abundantly rinsing in water. The process conditions were optimized to achieve arrays of nanoholes with diameter ranging from 30 to $200 \mathrm{~nm}$ and pitch from 100 to $300 \mathrm{~nm}$. Atomic force microscopy (AFM) analysis 
revealed holes depths between 7 and $10 \mathrm{~nm}$. GaN/InGaN NRs were grown on these patterned masks using an MBE system (Riber Compact 21). Details on the growth process and parameters can be found elsewhere [10]. The $\mathrm{Ga}$, In, and $\mathrm{N}$ fluxes were calibrated in equivalent (0001) GaN growth rate units for compact layers in $\mathrm{nm} / \mathrm{min}$, which are the standard units used in III-nitrides MBE growth diagrams [11]. The initial GaN segment was grown at a temperature of $900^{\circ} \mathrm{C}$, and the $\mathrm{Ga}$ and $\mathrm{N}$ fluxes were set to 16 and $5 \mathrm{~nm} /$ min, respectively. These conditions resulted in a highly-selective nucleation and growth of the GaN NRs inside the nanoholes. During the subsequent In incorporation (InGaN nano-disk), the temperature and Ga flux were lowered to $625^{\circ} \mathrm{C}$ and $4.3 \mathrm{~nm} / \mathrm{min}$, respectively. The In flux was set to $4.3 \mathrm{~nm} / \mathrm{min}$, while the $\mathrm{N}$ flux was increased to $14 \mathrm{~nm} / \mathrm{min}$. These conditions resulted in a $10-20 \mathrm{~nm}$ thick InGaN nano-disk, depending on the growth time (typically 5-10 min). For the growth of the final GaN cap, we used the same $\mathrm{Ga}$, N fluxes, and temperature as for the InGaN nano-disk growth, and closed the In shutter. The thickness of this GaN cap also varied with the growth time. The morphology of the masks and of the NRs was studied by AFM (Nanoscope III Multimode, Veeco Instr., USA) and scanning electron microscopy (SEM, CABL-9500C SEM, Crestec, Japan). The crystal quality and optical properties of the NRs were probed using transmission electron microscopy (TEM) and photoluminescence (PL), respectively.

\section{Results and discussion}

Fig. 1(a) and (b) shows typical Ti mask patterned by EBL/dry etch with different nanoholes geometries. The smallest holes, displayed in the AFM topography image in Fig. 1(a), had a diameter of $30 \mathrm{~nm}$. The pitch was varied between 100 and $300 \mathrm{~nm}$. Fig. 1(b) shows an SEM micrograph of a mask with $130 \mathrm{~nm}$ holes (diameter) and $350 \mathrm{~nm}$ pitch. Both these masks were obtained after a careful optimization of the Ti deposition, lithographic step, etching process, and post-etch cleaning [12].

Typical examples of NRs grown by PAMBE using these masks are shown in Fig. 2(a-c), respectively. In all cases, highly ordered and homogeneous GaN NRs grew with perfect selectivity within the nanoholes only, over areas of several hundred microns. Fig. 2(a) is a birds' eye view of the thinnest GaN NRs achieved on a mask with $30 \mathrm{~nm}$ holes. In particular, a border region between the hole-patterned and the unpatterned Ti mask is displayed.
Fig. 2(b and c) are TEM images of GaN NRs with an embedded InGaN nano-disk of about $12 \mathrm{~nm}$ in size, as derived directly from the TEM picture. In particular, Fig. 2(b) shows an array of these GaN/InGaN NRs that gives evidence for the growth direction perpendicular to the GaN substrate, thus in the (0001) direction. The conditions leading either to pyramidal or flat tops of the NRs are well investigated elsewhere [9,13]. Fig. 2(c) is a bright-field TEM of a single NR. In this picture the GaN bottom segment, embedded InGaN nano-disk, and top GaN cap are clearly distinguished. Moreover, there is evidence for InGaN growth in the region between the NRs (darker area, as indicated). The white layer observed between the surface (underneath the InGaN layer) and the GaN substrate consists of air, which is probably generated during the growth of the bottom GaN segment. At the high temperature used during the nitridation and GaN growth phase, the GaN substrate is expected to decompose. A possible explanation for the formation of an air void is that the Ga atoms diffuse to "feed" the growing GaN column, while the $\mathrm{N}$ atoms are expelled in gas form.

PL analysis of these pyramidal shape NRs yielded pertinent information only when performed on $\mathrm{GaN} / \mathrm{InGaN}$ NRs. Indeed, for pure GaN NRs the presence of the background spectrum from the $\mathrm{GaN}$ template renders the data interpretation difficult, especially due to the shortness of these NRs (typically $150-200 \mathrm{~nm}$ ). On $\mathrm{GaN} / \mathrm{InGaN}$ NRs samples as in Fig. 2(b), we could visually observe an intense light-blue emission at temperature between 12 and $300 \mathrm{~K}$, clearly arising from these NRs. Fig. 3(a and b) shows the PL spectra of GaN/InGaN NRs with a $\sim 20 \mathrm{~nm}$ thick embedded nano-disk. In both graphs two spectra are reported, that were recorded in two different regions within the patterned area, corresponding to different NRs diameter and pitch. The variation of the InGaN emission peak wavelength remains within a $10 \mathrm{~nm}$ accuracy, for all measured samples, thus proving the large-scale control of the average composition and thicknesses of the InGaN nano-disks. Quite systematically on this type of periodically patterned samples, we observe a modulation of a fairly-broad ( $0.15 \mathrm{eV}$ of full-width, typically) emission line, in the form of oscillations that may suggest different emission peaks. In fact, these are nothing but the result of interference effects induced by the periodicity of the nano-emitters. The images in Fig. 2(b and c) give evidence for parasitic growth of InGaN between the NRs, upon reduction of growth temperature, in addition to the growth that obviously occurred on the mask, away from the patterned regions. In fact, the periodic structures of the patterned regions induced an (a)

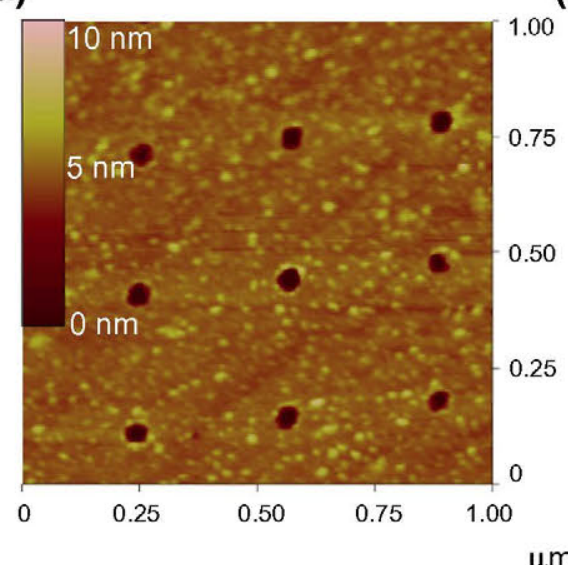

(b)

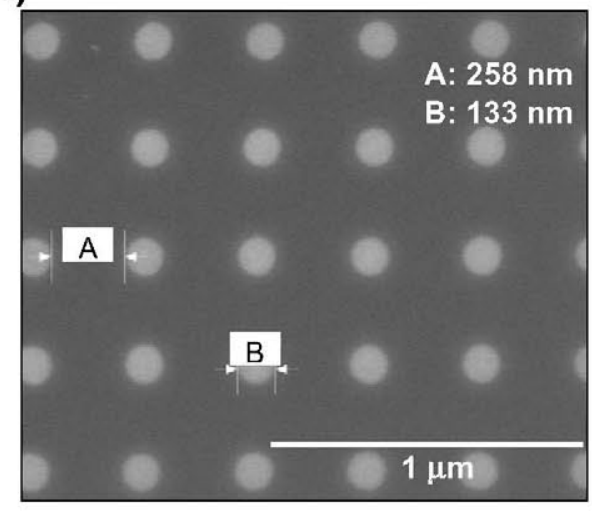

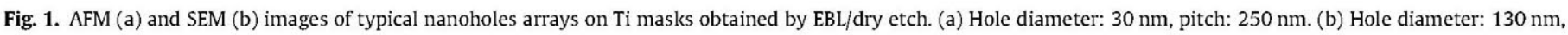
pitch: $350 \mathrm{~nm}$. 
(a)

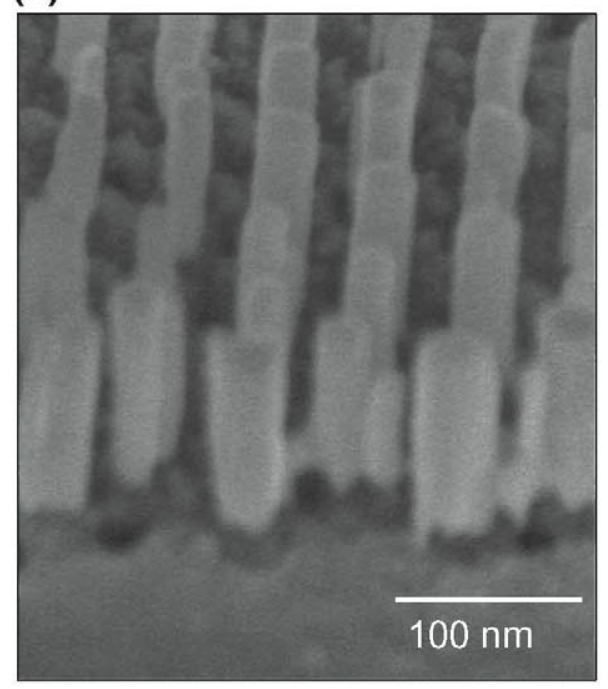

(b)

(c)
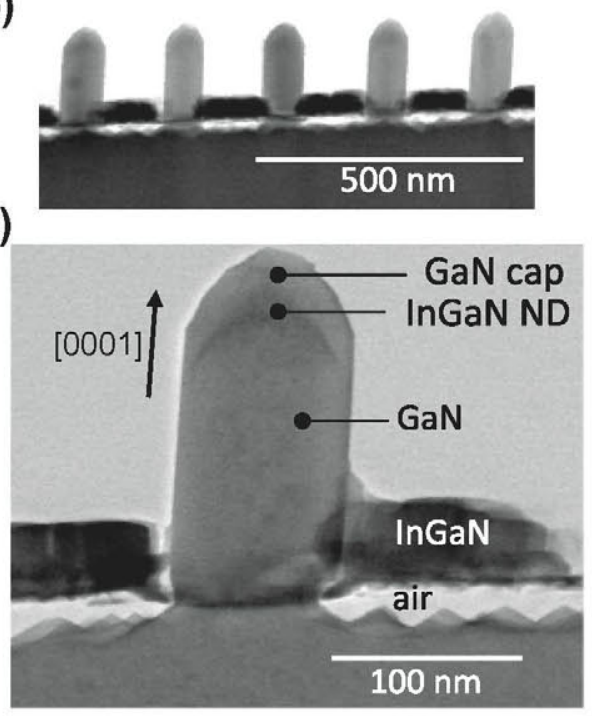

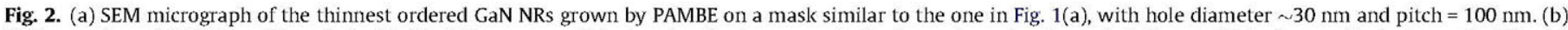

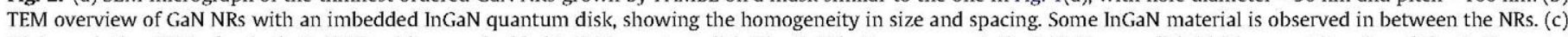

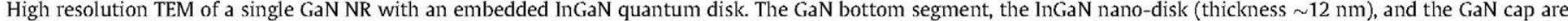

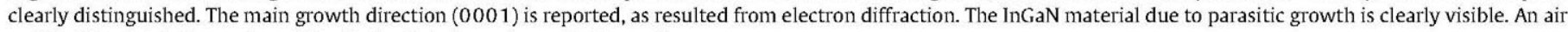
void is detected in the region of the GaN substrate underneath the surface.

(a)

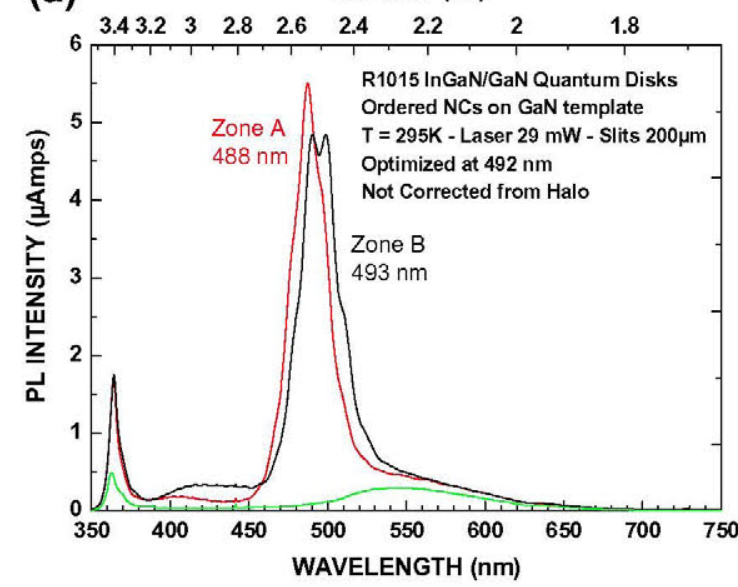

(b)

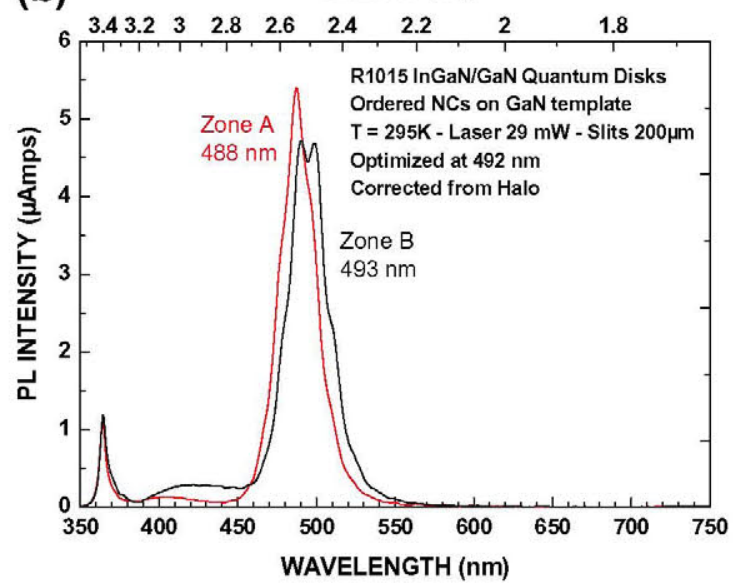

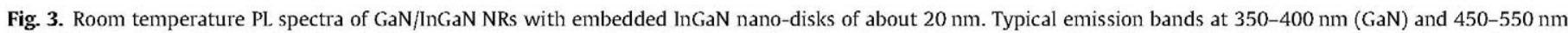

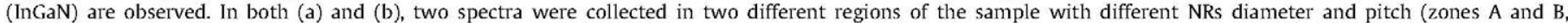

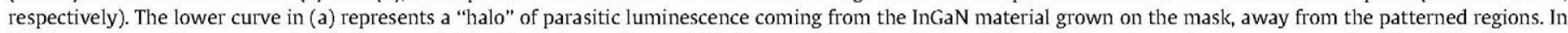
(b), the same spectra are reported, both corrected for the halo spectrum of (a). The wavelength emission of the InGaN peak was in all cases reproducible within 10 nm.

efficient in-plane scattering of the excitation laser, which excited secondarily a significant portion of the nearby unpatterned sample. This induced a "halo" of parasitic luminescence that we could analyze independently, by simply moving the laser spot away from the patterned regions. The result is shown by the green curve in Fig. 3(a), with no adjustment of intensities. Clearly, this weak, broad PL band centered at $550 \mathrm{~nm}$ can only arise from some parasitic growth of InGaN, and this experiment shows that a lot of it took place on the unpatterned mask, away from the periodic NR region. The result of a mere subtraction of this broad band from the overall PL is shown in Fig. 3(b), which we therefore consider "corrected from halo", as mentioned. This result indicates that PL from InGaN grown between the NRs, if any, yields a quite minor contribution to the overall signal. Temperature-dependent PL measurements allowed us to estimate and internal quantum efficiency
(IQE) at $300 \mathrm{~K}$ (the temperature of practical use for nano-emitters) of $18 \%$.

\section{Conclusions}

Carefully optimized e-beam/dry etch nano-patterning was performed on Ti films on GaN to create hole-patterned masks for the ordered growth of GaN/InGaN NRs by PAMBE. Highly reproducible and precise nanoholes arrays of various geometries were obtained over areas of microns. These patterned masks were used to fabricate highly ordered GaN/InGaN NRs. Pure GaN NRs nucleated selectively inside the nanoholes. When an InGaN nano-disk was embedded in the NRs, some parasitic growth took place in the regions between the NRs. However, PL analysis gives evidence for fully reproducible optical emission. 


\section{Acknowledgments}

This research was supported by a Marie-Curie fellowship within the 7th European Community Framework, PIEF-GA-2009-253085, National Projects CAM P2009/ESP-1503, MICINN-PLE2009-0023, MICINN-MAT-2008-04815, and by the UE CP-IP 228999-2 (SMASH) project.

\section{References}

[1] H. Sekiguchi, K. Kato, J. Tanaka, A. Kîkuchi, K. Kishino, Phys. Stat. Sol. (a) 205 (2008) 1067.

[2] W. Guo, M. Zhang, A. Banerjee, P. Bhattacharya, Nano Lett. 10 (2010) 3355.

[3] I.J. Hong, C.H. Chul-Ho Lee, A. Yoon, M. Kim, H.K. Seong, H.J. Chung, C. Sone, Y.J. Park, G.C. Yi, Adv. Mater. 23 (2011) 3284.
[4] H.P.T. Nguyen, K. Cui, S. Zhang, M. Djavid, A. Korinek, G.A. Botton, Z. Mi, Nano Lett. 12 (3) (2012) 1317

[5] M. Böberl, M.V. Kovalenko, G. Pillwein, G. Brunthaler, W. Heiss, Appl. Phys. Lett. $92(2008) 261113$.

[6] D.Y. Zhang, X.H. Zheng, L.J. Tang, J.R. Dong, H. Wang, H. Yang, IEEE Electron Dev. Lett. 31 (12) (2010) 1422

[7] J. Ristić, E. Calleja, A. Trampert, S. Fernández-Garrido, C. Rivera, U. Jahn, K.H. Ploog, Phys. Rev. Lett. 94 (2005) 146102.

[8] H. Sekiguchi, K. Kishino, A. Kikuchi, Appl. Phys. Express 1 (2008) 124002.

[9] H. Sekiguchi, K. Kishino, A. Kikuchi, Appl. Phys. Lett. 96 (2010) 231104.

[10] A. Bengoechea-Encabo, F. Barbagini, S. Fandez-Garrido, J. Grandal, J. Ristic, M.A. Sanchez-García, E. Calleja, U. Jahn, E. Luna, A. Trampert, J. Cryst. Growth 325 (2011) 89 .

[11] B. Heying, R. Averbeck, L.F. Chen, E. Haus, H. Riechert, J.S. Speck, J. Appl. Phys. $88(2000) 1855$.

[12] F. Barbagini, A. Bengoechea-Encabo, S. Albert, J. Martinez, M.A. Sanchez García, A. Trampert, E. Calleja, Nanoscale Res. Lett. 6 (2011) 632.

[13] S. Albert, A. Bengoechea-Encabo, P. Lefebvre, F. Barbagini, M.A. Sanchez-Garcia, E. Calleja, U. Jahn, A. Trampert, Appl. Phys. Lett. 100 (2012) 231906. 